\title{
129. Amphibian Blood Group Substances. IV
}

\author{
Biosynthesis of Blood Group B Substance \\ in Bull-frog's Gastric Mucosa
}

\author{
By Shigeru Yamamoto and Shoei ISEKI \\ Department of Legal Medicine, School of Medicine, \\ Gunma University, Maebashi \\ (Comm. by Tanemoto FuruhatA, M. J. A., June 12, 1971)
}

Papers on biosynthesis of blood group substances of $\mathrm{ABO}$ and Lewis systems have been recently issued, most of which are forcussing on in vitro transfer of group-specific sugars from corresponding sugar nucleotides to oligosaccharides or red cells. However, in vitro experiments are inconceivable to be sufficient to elucidate mechanism of biosynthesis of high molecular weight of blood group substances in living matter.

As authors were able to give evidence that considerable amounts of uridine diphosphate galactose (UDP-galactose) supposed to be a galactose donor is synthesized in vivo in livers and in vitro in gastric mucosa of bull-frogs, ${ }^{1)}$ we shall offer here further results on in vivo and in vitro biosynthesis of blood group B substance present in the frog's stomachs. ${ }^{2}$

Materials and methods. 1. Incorporation of ${ }^{14}$ C-galactose. Methods of in vivo incorporation of galactose-1- ${ }^{14} \mathrm{C}$ (The Radiochemical Centre, specific activity of $55.7 \mathrm{mCi} / \mathrm{mM}$ ) into bull-frog's hearts and incubation condition of the gastric mucosa homogenates with the same species of isotope were the same as those described in the preceding paper. ${ }^{1)}$

2. Measurement of radioactivity. The radioactivity of the gastric blood group substance was measured by liquid scintillation counter (Tricarb), after solubilization with $1 \mathrm{ml}$ of Hyamine $10 \mathrm{X}$ prior to add dioxane solvent employed in the previous experiment. ${ }^{1)}$

3. Isolation and preparation of gastric blood group substance. The stomachs were immediately cut out at a given time after injection of ${ }^{14} \mathrm{C}$-galactose, washed several times with cold saline solution, dipped into absolute ethyl alcohol, and stood at $2^{\circ} \mathrm{C}$. Absolute ethyl alcohol was replaced every day for one week. Thereafter the stomachs were dried by immersing in ethyl ether, followed by submitting to preparation of gastric blood group substances as described in elsewhere. ${ }^{2)}$ Likewise, the gastric blood group substances were prepared 
from the sediment fraction which was obtained after sugar nucleotides were separated by centrifugation from the incubation mixture of the gastric mucosa with ${ }^{14} \mathrm{C}$-galactose.

4. Analysis of component sugar. Paper chromatography and detection of component sugars as well as assay for $\alpha$-D-galactosyl residue by $\alpha$-D-galactosidase preparation from Clostridium sporogenes Maebashi were described in the two previous papers. ${ }^{3,4)}$

Results. 1. In vivo incorporation of ${ }^{14} C$-galactose into gastric blood group substance. Incorporation of ${ }^{14} \mathrm{C}$-galactose was examined on two kinds of blood group-active fractions prepared from the gastric mucosa which were taken out at a given time after injection of ${ }^{14} \mathrm{C}-$ galactose into bull-frog's hearts. Although phenol-soluble and 50\% ethyl alcohol precipitates is known to be higher than phenol-insoluble

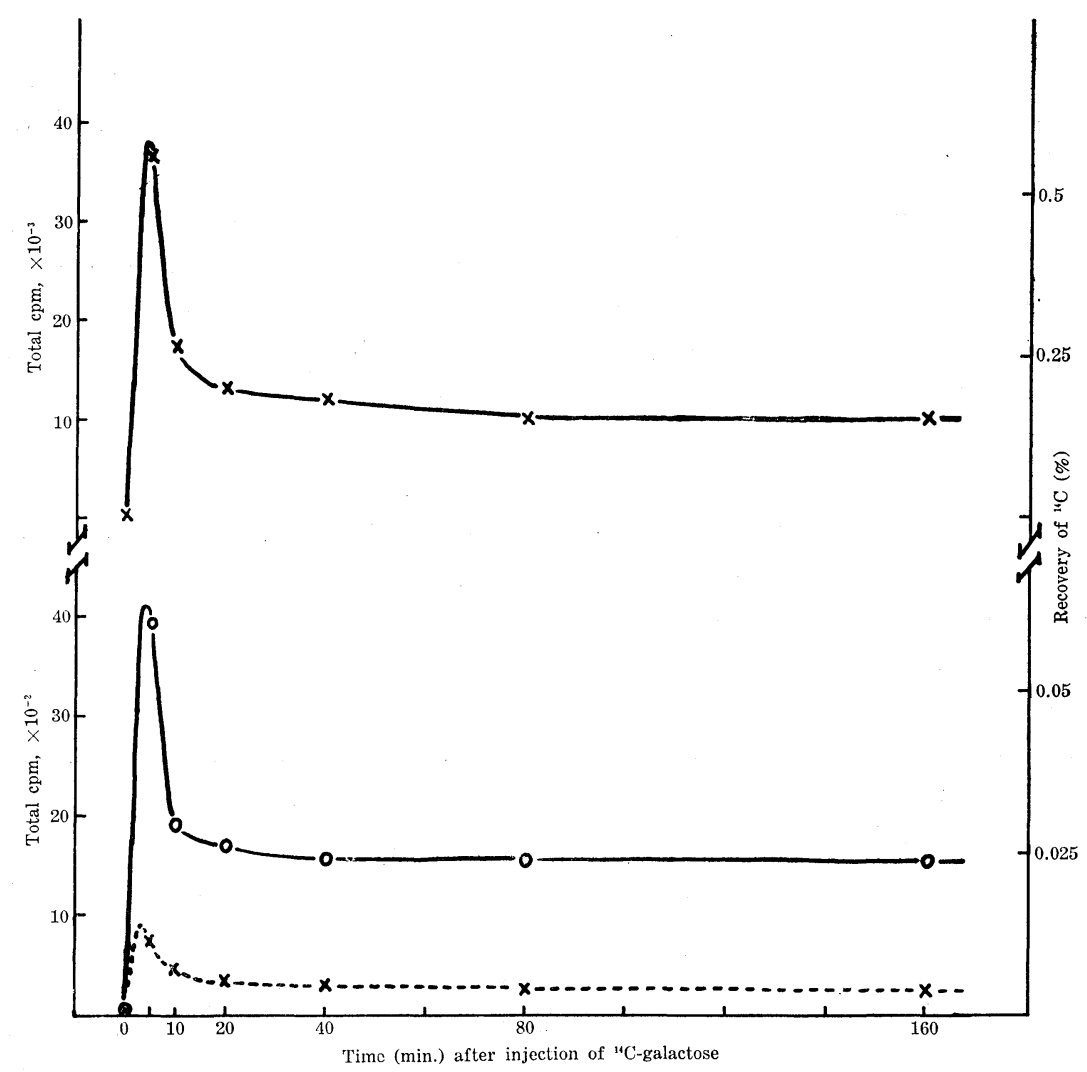

Fig. 1. Incorporation of ${ }^{14} \mathrm{C}$-galactose into gastric blood group substances.

$-X-\times-:$ Phenol-soluble and 50\% ethanol ppt./5 frogs.

$-\times---\times-$ : Phenol-soluble and $50 \%$ ethanol ppt./10 mg.

$-\bigcirc-O-:$ Phenol-insoluble fraction $/ 5$ frogs. 
fraction in blood group B activity, ${ }^{2)}$ both fractions are labeled with ${ }^{14} \mathrm{C}$ within short time, as seen in Fig. 1.

The incorporation of ${ }^{14} \mathrm{C}$ reaches the maximum not later than 5 minutes after injection of the isotope, rapidly decreasing thereafter till 10 minutes and holds a constant level, irrespective of nature of blood group activity. However, relative rate of ${ }^{14} \mathrm{C}$-incorporation is always about ten times higher in the phenol-soluble than in the phenolinsoluble fraction throughout of time elapsed.

The radio-active phenol-soluble fraction resulted was treated with $\alpha$-D-galactosidase from $\mathrm{Cl}$. sporogenes Maebashi to determine whether the radioactivity was introduced by the form of galactose or by the other forms. $91 \%$ and $85 \%$ of total radioactivity was shown to be released from specimens prepared from 5 minutes and 80 minutes, respectively, after injection of ${ }^{14} \mathrm{C}$-galactose. Paper chromatography of the enzymic incubation mixture also showed that the radioactivity is located on galactose area, so that the blood groupactive fractions are known to be labeled with ${ }^{14} \mathrm{C}$ in the $\alpha$-galactosyl residues for the most part. Hemagglutination inhibition test was performed on blood group B activity of the radioactive fraction, too. $B$ activity was shown to be enhanced two or three times higher in the fraction with the highest radioactivity than in control, though $B$ activity in the latter is considerably high by nature.

2. Biosynthesis of blood group B substance by gastric mucosal homogenates. Though evidence was given that blood group B substance is formed in vivo in the gastric mucosa, it is still doubtful whether the blood group substance is transported into the gastric tissues after formation in other organs or formed by an enzyme, $\alpha$-D-galactosyl transferase present in the stomach. From the incubation mixture of the gastric mucosal homogenates with ${ }^{14} \mathrm{C}$-galactose were isolated and purified two kinds of blood group-active fractions, phenol-soluble and $50 \%$ ethyl alcohol precipitates and phenol-insoluble fraction. As shown in Fig. 2, ${ }^{14} \mathrm{C}$ is incorporated with remarkably high speed into these blood group-active fractions and reaches the maximum at about 40 minutes, without reduction of the radioactivity in longer period. The amount of radioactivity is about fifteen times larger in the phenol-soluble than in the phenol-insoluble fraction, which depends on relative yield between the two fractions, larger in the former and smaller in the latter. The in vitro experiment also indicates that blood group B substance is synthesized under function of $\alpha$-D-galactosyl transferase inherent in the gastric mucosa, which transfer galactosyl residue of UDP-galactose synthesized from galactose in the same tissues into an endogeneous precursor (s) of B sub- 


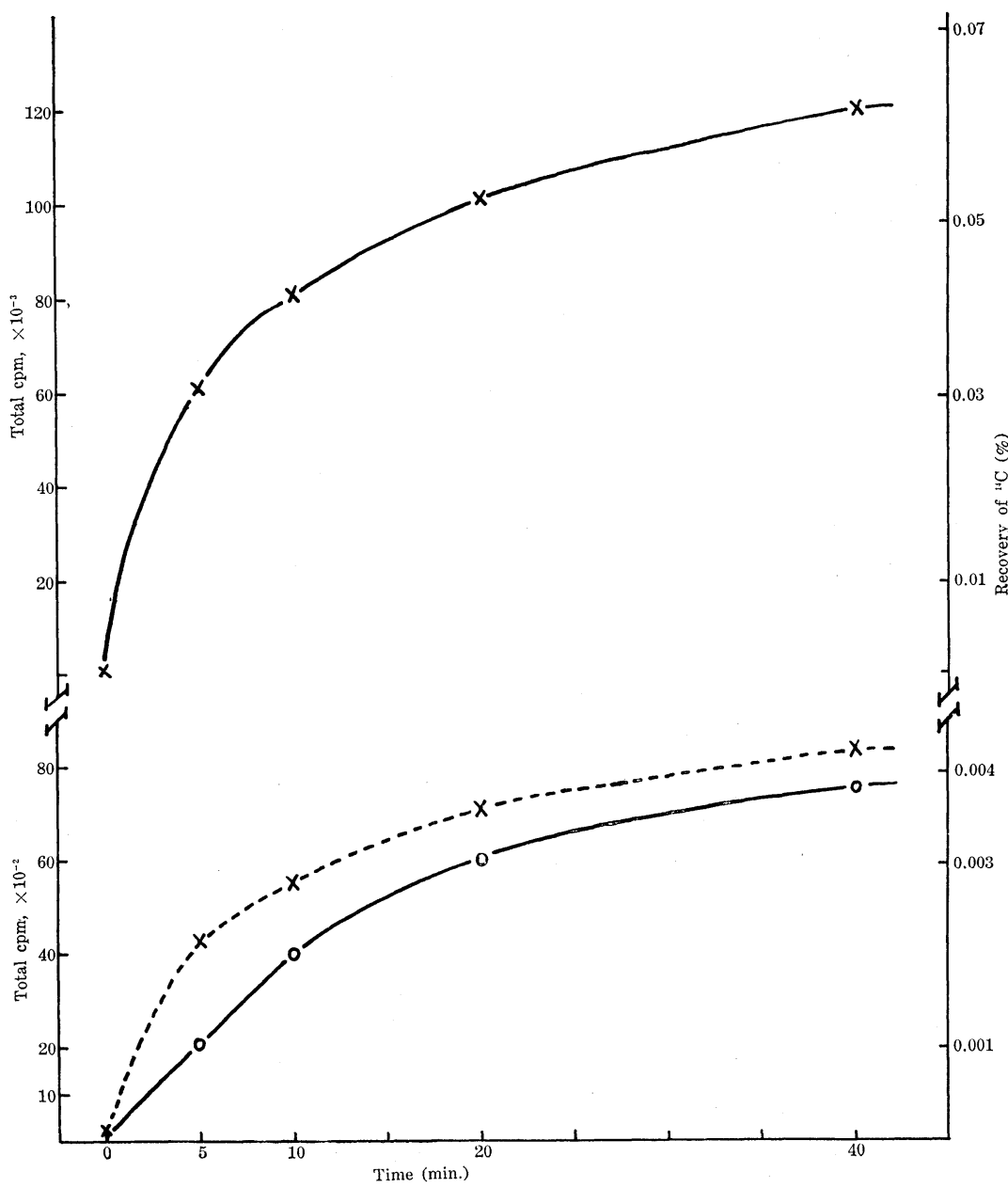

Fig. 2. Time course of in vitro biosynthesis of gastric blood group substance.

$-X-X-:$ Phenol-soluble and 50\% ethanol ppt./8 frogs.

$-X---X-$ : Phenol-soluble and $50 \%$ ethanol ppt./10 mg.

$-\bigcirc-\bigcirc-$ P Phenol-insoluble fraction/8 frogs.

stance.

Discussion. Blood group B substance is synthesized, rapidly beyond expectation, in the gastric mucosa, the maximum amount of which reaches within 5 minutes after injection of ${ }^{14} \mathrm{C}$-galactose into the bull-frog's heart. As compared with it, UDP-galactose as well as UDP-glucose formed in vivo in the liver reached the maximum amount in 10 to 20 minutes after injection of the isotope ${ }^{1)}$ so that UDPgalactose required for the synthesis of $B$ substance in the stomach is supposed to be formed in the same organ rather than supplied 
from the liver. Thereupon, it is difficult to explain a phenomenon that B substance formed in the vivid stomachs reduces as soon as it reaches the maximum level. Allowing an assumption, bull-frog's stomachs may release an excess of blood group substance from the mucosal linings into the gastric juice after maturation or over-production.

On the other hand, biosynthesis of B substance in the incubation of the gastric mucosal homogenates with galactose proceeds with time incubated.

Results obtained here and in the preceding paper ${ }^{1)}$ confirm that $\alpha$-D-galactosyl transferase with potent activity is present in the gastric mucosa of bull-frogs, which is able to use UDP-galactose as a galactosyl donor and high molecular weight of precursor (s) of B substance as acceptor.

The enzyme relating to biosynthesis of $\mathrm{B}$ substance is reported to be present in $\mathrm{B}$ or $\mathrm{AB}$ type of human gastric mucosa and submaxillary gland, ${ }^{5)-7)} \mathrm{B}$ or $\mathrm{AB}$ type of mother milk, ${ }^{8)}$ and gastric mucosa of baboon and rabbit,, ${ }^{5,6)}$ transferring galactose from UDPgalactose to oligosaccharide acceptors or erythrocytes.9) However, it is still unclear whether these enzyme preparations mentioned above act on high molecular weight of acceptors as they do on low molecular oligosaccharides. $\alpha$-D-Galactosyl transferase found in bull-frogs seems to be similar, on substrate specificity, to that prepared recently by Poretz and Watkins ${ }^{10)}$ from human stomach and submaxillary gland with B character.

Acceptor specificity of phenol-soluble or phenol-insoluble fractions as precursor of $\mathrm{B}$ substance as well as relationship between blood group synthesizing enzymes and seasonal variation of $\mathrm{B}$ activity $^{2)}$ remain to be investigated.

Conclusion. Incorporation of ${ }^{14} \mathrm{C}$-galactose into precursor $(\mathrm{s})$ of blood group B substance endogeneously present in bull-frog's gastric mucosa was recognized to reach the maximum within 5 minutes after injection of it into the heart and reduce thereafter to a given level. It was also known that the gastric phenol-soluble or phenol-insoluble fractions with $\mathrm{B}$ activity are rapidly synthesized in incubation of the gastric mucosa homogenates with ${ }^{14} \mathrm{C}$-galactose by function of $\alpha$-Dgalactosyl transferase.

\section{References}

1) Yamamoto, S., and Iseki, S.: Amphibian blood group substances. III. Biosynthesis of uridine diphosphate galactose in bull-frog's livers and stomachs. Proc. Japan Acad., 47(6), 567-572 (1971). 
2) Yamamoto, S., and Iseki, S.: Amphibian blood group substances. I. ABH blood group activities of erythrocytes and gastric mucosa from Xenopus and Rana. Proc. Japan Acad., 45(10), 974-979 (1969).

3) ——: Amphibian blood group substances. II. Chemical components of gastric blood group substances from Xenopus and Rana. Proc. Japan Acad., 46(2), 191-196 (1970).

4) Yamamoto, S., Fujisawa, K., and Iseki, S.: Chemical effects of purified A- and B-decomposing enzymes on blood group A and B substances. Gunma J. Med. Sci., 11(4), 248-258 (1962).

5) Zinderman, D., Gompertz, S., Smith, Z. G., and Watkins, W. M.: Glycosyl transferases in mammalian gastric mucosal linings. Biochem. Biophys. Res. Commun., 29(1), 56-61 (1967).

6) Race, C., Zinderman, D., and Watkins, W. M.: An $\alpha$-D-galactosyl transferase associated with the blood group B character. Biochem. J., 107(5), 733-735 (1968).

7) Race, C., and Watkins, W. M.: Properties of an $\alpha$-D-galactosyl transferase in human tissues obtained from blood group B donors. Biochem. J., 114(4), 86 p (1969).

8) Kobata, A., Grollman, E. F., and Ginsburg, V.: An enzymatic basis for blood type B in human. Biochem. Biophys. Res. Commun., 32(2), 272-277 (1968).

9) Shenkel-Brunner, H., and Tuppy, H.: Enzymes from human gastric mucosa conferring blood-group A and B specificities upon erythrocytes. Eur. J. Biochem., 17(2), 218-222 (1970).

10) Poretz, R., and Watkins, W. M.: unpublished, in Blood and Tissue Antigens (edited by Aminoff, D.). Academic Press, New York and London, 441-459 (1970). 